\title{
Driver-initiated Tesla Autopilot Disengagements in Naturalistic Driving
}

\author{
Alberto Morando \\ MIT AgeLab, Massachusetts Institute of Technology \\ morando@mit.edu \\ Bruce Mehler \\ MIT AgeLab, Massachusetts Institute of Technology \\ bmehler@mit.edu
}

\begin{abstract}
We quantify the time-course of glance behavior and steering wheel control level in driver-initiated, non-critical disengagements of Tesla Autopilot (AP) in naturalistic driving. Although widely used, there are limited objective data on the impact of AP on driver behavior. We offer insights from 19 Tesla vehicle owners on driver behavior when using AP and transitioning to manual driving. Glance behavior and steering wheel control level were coded for 298 highway driving disengagements. The average proportion of off-road glances decreased from $36 \%$ when AP was engaged to $24 \%$ while driving manually after AP disengagement. Most of the offroad glances before the transition were downward and to the center stack (17\%). Lastly, in 33\% of the events drivers were not holding the steering wheel prior to AP disengagement. The study helps begin to enhance society's understanding, and provide a reference, of real-world AP use.
\end{abstract}

\section{CCS CONCEPTS}

- Human-centered computing $\rightarrow$ Empirical studies in $\mathrm{HCI}$.

\section{KEYWORDS}

Automation, driver behavior, attention, takeover, transition of control

\section{ACM Reference Format:}

Alberto Morando, Pnina Gershon, Bruce Mehler, and Bryan Reimer. 2020. Driver-initiated Tesla Autopilot Disengagements in Naturalistic Driving. In 12th International Conference on Automotive User Interfaces and Interactive Vehicular Applications (AutomotiveUI '20), September 21-22, 2020, Virtual Event, DC, USA. ACM, New York, NY, USA, 9 pages. https://doi.org/10.1145/ 3409120.3410644

\section{INTRODUCTION}

Tesla Autopilot (AP) is a bundle of vehicle automation features designed as assisted driving systems to keep the vehicle in the lane (Autosteer) and at a safe distance from other traffic (trafficaware cruise control, T-ACC). AP is classified as a SAE Level 2

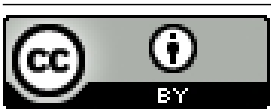

This work is licensed under a Creative Commons Attribution International 4.0 License. AutomotiveUI '20, September 21-22, 2020, Virtual Event, DC, USA

(c) 2020 Copyright held by the owner/author(s).

ACM ISBN 978-1-4503-8065-2/20/09.

https://doi.org/10.1145/3409120.3410644

\author{
Pnina Gershon \\ MIT AgeLab, Massachusetts Institute of Technology \\ pgershon@mit.edu \\ Bryan Reimer \\ MIT AgeLab, Massachusetts Institute of Technology \\ reimer@mit.edu
}

automated driving feature [34] and, as such, it requires active driver supervision. Tesla AP is considered to be one of the most capable systems commercially available [4, 12]. Recent studies indicate that Tesla owners use AP frequently and their confidence with the system increases the more they use it [7-9].

Automation, such as AP, enhances comfort for many drivers and has been associated with reports of reduced driving demand $[6,28]$, but the safety benefits of these systems are still debated as there is a potential for over-reliance [12, 28, 31, 35]. As automation improves and failures become rarer, drivers may tend to forgo their monitoring role: be less attentive (more time eyes off-road) and relax direct control (hands off-wheel) [1, 9, 42]. Perhaps, if automation handles most driving scenarios smoothly, drivers may believe that the automation can handle any situations and begin to over trust the system, impacting their capability to respond to failures [29, 42]. These unintended behavioral adaptations [36] are often detailed in social media posts and news articles, but are less understood from the scientific perspective. More research is needed to understand the benefits and limitations of assisted driving systems that can support a comprehensive safety benefit analysis.

A growing body of research is concerned with the potential for detrimental effects of automation on driver behavior (e.g., lower awareness of the surrounding traffic [28]) and performance (e.g., lower quality of evasive maneuvers [22]). To date, most of the knowledge about driver behavior with advanced automation is based on simulator experiments $[6,28,33]$ or relatively small scale on-road studies $[1,9,10,42]$. Despite this growing body of empirical work, little is known about the actual use of assistive driving systems in real-world driving. Several high-profile crashes with AP [e.g., 26, 27] have highlighted the need to further research driver behavior when using automation systems.

With assistive driving systems, such as AP, drivers are expected to maintain visual attention to events on the roadway and be ready to regain control of the vehicle. Otherwise, drivers may fail to promptly detect and respond to critical situations or system malfunctions [22, 42]. A survey by Dikmen and Burns [7] reported that about $60 \%$ of participants experienced sporadic, erratic AP behavior (e.g., lane detection or road departure issues). Similarly, Endsley [9] reported experiencing AP malfunctions in $30 \%$ of the trips. Although relatively rare, if drivers are not ready to regain control in these situations, consequences can be tragic [e.g., 26, 27].

Tesla's safety reports suggest that many consumers are safely utilizing AP [38]. While AP does not monitor the degree of driver attention to the road, it does use a torque-based hands-on-wheel 
sensing system as a mechanism to monitor driver engagement. The owner's manual states that the system detects the hands by recognizing light resistance as the steering wheel turns, or by manually turning the steering wheel lightly [37]. If the hands are not detected, AP will display a warning on the instrument cluster; if the warning is repeatedly ignored, the car will begin to slow down to a complete stop [37]. Tesla AP has long been criticized for failing to ensure that drivers are paying attention to the road and are not misusing the system [13, 20,32]. However, the degree to which drivers maintain attention to the road and keep their hands on the steering wheel during AP use has yet to be substantiated in the scientific literature.

The aim of this paper is to provide novel insights on how driver behavior changes with the use of automation and during transitions of control. Driver behavior was examined in terms of eye glance behavior and steering wheel control level before, during, and immediately after AP disengagement. The uniqueness of this paper comes from the large naturalistic driving dataset of Tesla owners, capturing AP use in real world traffic. Despite the focus on a specific implementation of automation, these findings can inform improvements in the design of assistive and automated vehicle technology as a whole, by enhancing knowledge on how drivers leverage automation under real-world operating conditions.

\section{METHODS}

\subsection{Data source}

Data were drawn from the ongoing MIT Advanced Vehicle Technology (MIT-AVT) naturalistic driving study [14]. Starting in 2016, the project began following owners of Tesla Model S/X driving their vehicles in real traffic for periods of a year or more. While a predominance of the vehicles are garaged in the greater Boston area, several owners are from other New England states and trips are frequently logged outside of New England. Vehicles are equipped with the RIDER (Real-time Intelligent Driving Environment Recording) data acquisition system [14]. The system continuously collected data from: (i) the controller area network (CAN) bus to determine vehicle kinematics, driver interaction with the vehicle controllers, and the state of in-vehicle automation systems (e.g., AP state), (ii) a Global Positioning System (GPS) to record mileage and location; (iii) three $720 \mathrm{p}$ video cameras that continuously recorded (30 fps) the driver's posture, driver's face, the forward view of the vehicle, and the instrument cluster. The camera recordings were used to extract features related to driver behavior and driving environment. The foundation for CAN data discovery leveraged open source information (e.g., [3]). It was then extended through further investigation, discovery, and validation. CAN data validation was done by the first author frame-by-frame on a subset of the data. Part of the validation process consisted of ensuring that the CAN signals for the Autopilot state corresponded to what was displayed on the instrument cluster.

\subsection{Data reduction and measures}

The MIT-AVT activity is an on-going driving data collection effort focused on developing an understanding of system performance and how drivers adapt to, use (or do not use), and behave with advanced vehicle technologies including a wide range of assistive automated driving features. This analysis started with a pool of nearly $500 \mathrm{k}$

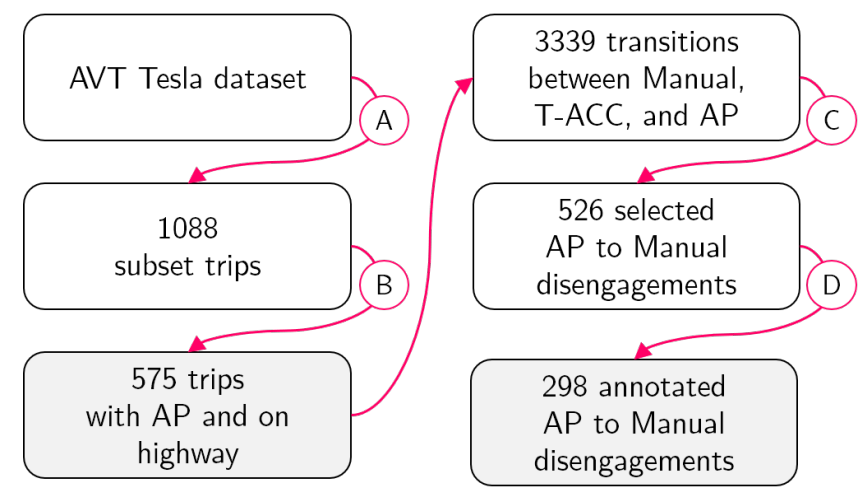

Figure 1: Data selection workflow. Each block represents an intermediate data sample starting from the full naturalistic database. Samples used in the results section are shaded. Each processing step is marked by a circle letter. Details on the operations are in the text.

miles of driving in the AVT Tesla dataset sampled based on miles driven and use of AP of each driver (i.e., driving exposure; step $A$ in Figure 1). We obtained the subset, first, by filtering out trips with known low-quality data, at night (cameras without infrared), or unusually long (above 120 miles). Second, we calculated a weight factor $w$ for each driver as the proportion of miles driven by that driver to the total miles driven across the full dataset. Third, as we aimed for about 1000 total trips, we determined the desired number of trips for each driver as $w \times 1000$. Finally, out of the desired number of trips per driver, we sampled $75 \%$ of the trips when AP was used at least once and $25 \%$ without any AP use. In the end, the subset included a total of 1088 trips (about $21 \mathrm{k}$ miles) by 27 drivers ( 8 females) with an average age of 48 years $(\mathrm{SD}=13$ years), and 21 cars. Fourteen cars were equipped with AP version 1 (they accounted for $87 \%$ of the trips); five were equipped with AP version 2 (they accounted for $12 \%$ of the trips); 2 were not equipped with $\mathrm{AP}$ (they accounted for $1 \%$ of the trips). AP version 2 is an upgrade to version 1 in terms of computing power and sensors. Then, we further selected 575 out of 1088 trips in AP-enabled vehicles that drove at least partly on a limited access highway (as identified by Open Street Map as motorway), which is the core operational design domain for AP [37] (based on GPS information for mapmatching; step $B$ in Figure 1). These 575 trips contained a total of 3339 transitions between manual driving, T-ACC, and AP.

Among all control transitions in highway driving, we selected non-critical, driver-initiated AP disengagements to manual control (henceforth referred as AP disengagement; step $C$ in Figure 1). We investigated this type of disengagements-in contrast to many studies that focused on critical, system-initiated transitions-because they are the most common and they likely provide a reference on aspects of normative driver behavior with AP [see also 11]. The AP disengagements were selected according to the following criteria: (i) AP was used for at least $30 \mathrm{~s}$ prior the disengagement without being overridden by accelerating or steering; (ii) There were at least $10 \mathrm{~s}$ of manual driving after disengagement; (iii) Road class was highway; (iv) Speed was above $30 \mathrm{mph}$; (v) Longitudinal acceleration was less than $-3 \mathrm{~m} / \mathrm{s}^{2}$; (vi) no advanced driver assistance system 
(ADAS) warnings were issued (e.g., forward collision warning). We obtained 526 AP disengagements.

Out of the 526 selected AP disengagements, 228 were excluded because the driver's eyes or hands were not visible (e.g., the camera was misaligned or the driver was wearing sunglasses; step $D$ in Figure 1). Eventually, a total of 298 AP disengagements were analyzed. This set of AP disengagements was from 19 drivers (4 females), across 198 unique trips, with an average of 16 AP disengagements per driver $(\min =3$ and $\max =93)$. Most of the events $(n=268)$ were with AP version 1. For each of the 298 AP disengagements, we selected a $30 \mathrm{~s}$ driving segment $(20 \mathrm{~s}$ before and $10 \mathrm{~s}$ after the disengagement) that could be fully annotated for glance location and steering wheel control level. The time course of glance and steering wheel control behavior was analyzed with respect to three intervals. The first and last $5 \mathrm{~s}$ provide an estimate of steady-state driver behavior with and without AP; The $5 \mathrm{~s}$ around the disengagement highlighted the changes in driver behavior during transition phase.

2.2.1 Glance behavior. Each event was analyzed by experienced coders, who viewed the $30 \mathrm{~s}$ video segments and employed a systematic frame-by-frame glance coding protocol. Each event was coded once by a single coder. Inter-rater reliability was assessed qualitatively on a small collection of videos and it was deemed satisfactory (i.e., coders assigned the same label within a few frames). Glances were classified according to the following categories: (i) Road (any glance directed outside the windscreen); (ii) Instrument cluster (any glance to the instrument cluster or the steering wheel region); (iii) Down center stack (any glance to the center stack, the in-car multimedia touch-screen, or down when looking, for example, at a smartphone); (iv) Windows mirrors (any glance to the side windows or mirrors; this category may also include glances towards a passenger sitting next to the driver but out of the camera's field of view); and (v) Rearview mirror. When a glance did not fall under the categories mentioned above (e.g., over the shoulder glance or eyes were closed), it was coded as Other. If the driver's eyes were temporarily not visible, glances were coded as Not available. Lastly, following ISO 15007-1:2014, a single glance consisted of the transition time toward an area of interest and the subsequent dwell time on that area. At any given timestamp, we computed the proportion of each glance location across the events; this resulted in $(30 \mathrm{~s}$ x 30 $\mathrm{Hz}=) 900$ values of aggregated glance location proportion.

2.2.2 Steering wheel control. Drivers' steering wheel control was manually coded frame-by-frame based on the observed hand positioning on the steering wheel. Similar to De Waard et al. [5], the coding protocol included four levels of steering control (Figure 2): (i) High steering control (when both hands were on the steering wheel at the optimal 3-9 o'clock position-the pose classically learned in driving school); (ii) Medium steering control (when the driver had only one hand at the 3-9 o'clock position, and the other one was elsewhere on the steering wheel); (iii) Low steering control (when both driver's hands were not at the 3-9 o'clock position, or when only one hand was placed on the steering wheel regardless of its position); (iv) None (when there was no hand grabbing the steering wheel; for example, if the driver had only a finger on the steering wheel it was coded as None); (v) the Other category was used in cases where the steering control level did not fit the categories listed above (e.g., hands hovering, steering with a knee); lastly (vi) the Not
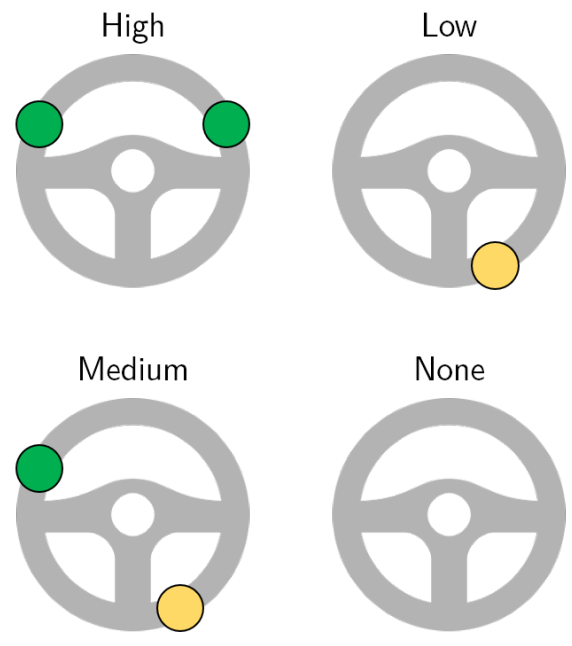

Figure 2: Examples of steering wheel control levels. Circles represents hands on the steering wheel. If the hand is positioned on an optimal control position (i.e., 3-9 o' clock) it is highlighted in green. Otherwise, it is highlighted in yellow.

available label was used when the driver's hands were momentarily not visible or in poor video quality. Then, at any given timestamp, we computed the proportion of steering wheel control levels across the events; this resulted in $(30 \mathrm{~s} \times 30 \mathrm{~Hz}) 900$ values of aggregated steering wheel control level proportions.

\section{RESULTS}

Within the initial subset of 1088 trips, highway driving accounted for about $13 \mathrm{k}$ miles. Within the periods of highway driving, AP was used for about $9 \mathrm{k}$ of the miles driven (Figure 3), which is about $70 \%$ of the distance for which AP was available. On highways, AP disengagements to manual driving accounted for $28 \%$ of all transitions and they were the most common disengagement (64\%; Figure 3). The typical way to disengage AP is by pushing the AP stalk once or by braking (overriding the steering would disengage Autosteer but leave T-ACC on) [37]. Most of the AP disengagements were in anticipation of taking an exit ramp, of a lane change, or of a construction zone. Five AP disengagements were system-initiated (takeover requests, [37]). A takeover request is issued in those situations in which AP is unable to steer the vehicle (e.g., sensor malfunctions, poor lane markings, adverse weather, or too sharp turns [37]).

\subsection{Glance behavior}

Steady-state glance behavior. Table 1 shows the aggregated steadystate glance behavior with and without AP active. With AP the proportion of on-road glances was lower than in manual driving. The lower proportion of on-road glances was mainly counterbalanced by the increase of glances down and to the center stack with AP compared to manual driving. The change in proportion for the remaining glance categories was, instead, minimal. An alternative way to look at the results in Table 1 is the ratio of off-road glances to on-road glances. With AP the ratio was 1:1.8 and this ratio shifted 


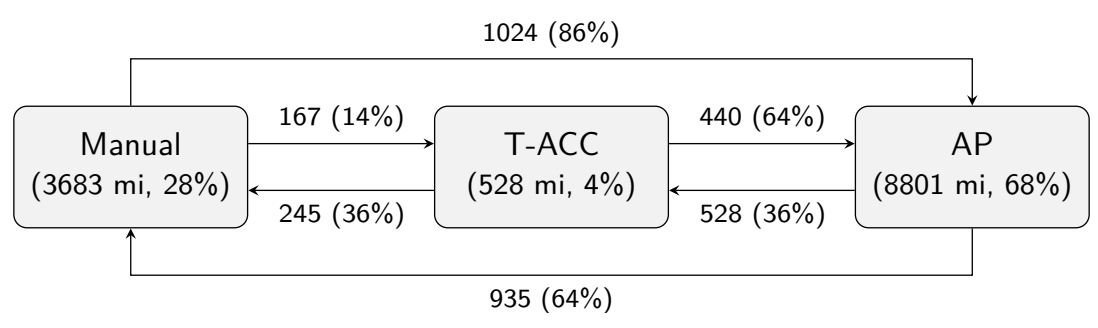

Figure 3: State transition diagram for highway driving based on 3339 transitions from 575 trips. Each arrow is annotated with the number of transitions and the percentage with respect to all transitions exiting the state. The transition from manual driving to traffic aware cruise control (T-ACC) requires T-ACC to be available and the driver to pull the cruise control stalk once. Transitioning to AP requires Autosteer to be available and the driver to pull the cruise control stalk twice. The transition to AP is possible from either the manual or T-ACC state. T-ACC or AP can be disengaged by pushing the cruise control stalk or by braking. The transition from AP to T-ACC is possible only by overriding steering [37]. Each state reports the aggregated distance (in miles and percent of the total) traveled in that state.

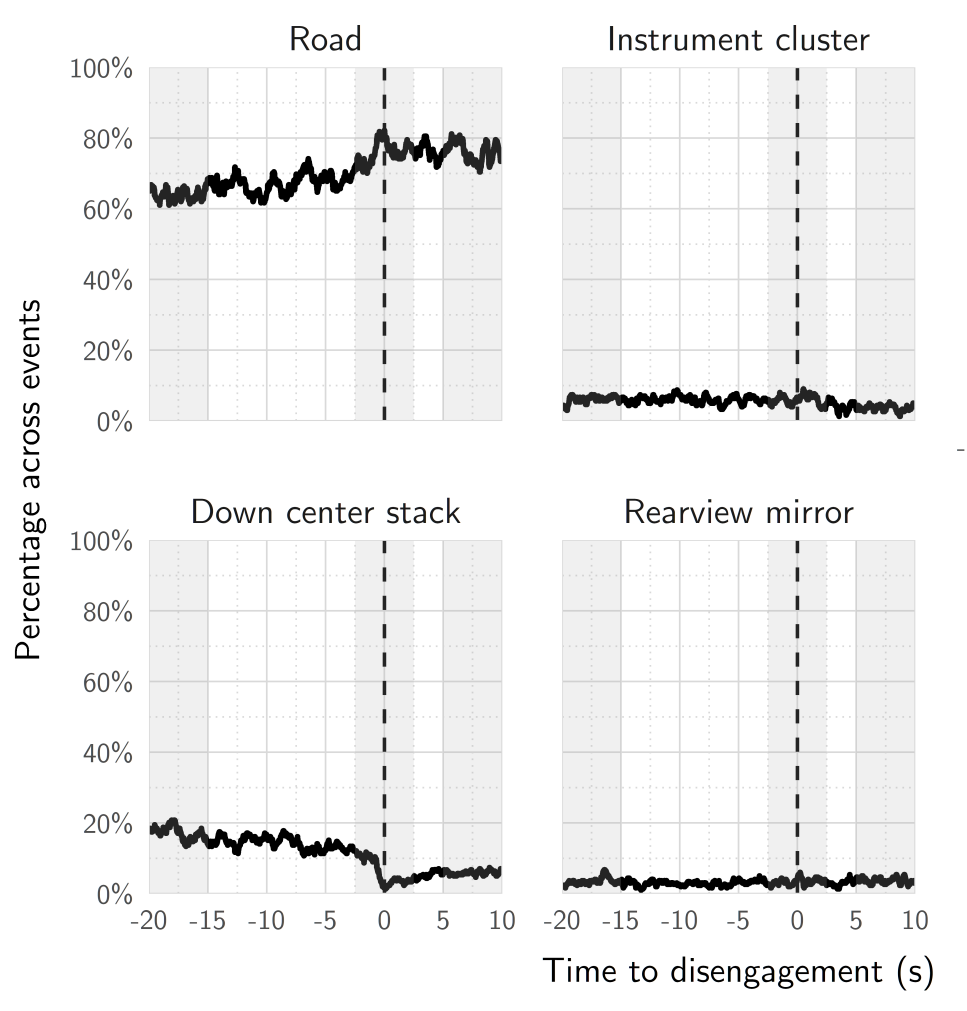

Figure 4: Proportion of glance locations at any given time during the events under analysis. AP disengagement is at $0 \mathrm{~s}$, marked with black dashed line. The shaded bands indicate the interval of estimated steady-state driving with Autopilot (AP; i.e., the $-20 \mathrm{~s}$ to $\mathbf{- 1 5} \mathrm{s}$ interval before the disengagement), the transition phase from AP to manual driving (i.e., the $-2.5 \mathrm{~s}$ to $2.5 \mathrm{~s}$ interval around the disengagement) and the interval of estimated steady-state manual driving (i.e., the $5 \mathrm{~s}$ to $10 \mathrm{~s}$ interval after the disengagement).

to $1: 3.2$ under manual control. Overall, this represents a $20 \%$ percent increase in visual attention to the road when driving manually, or a $50 \%$ increase in visual attention away from the road when driving with AP.
Glance behavior during the transition. Figure 4 shows the timecourse of glance behavior from $20 \mathrm{~s}$ before to $10 \mathrm{~s}$ after the AP disengagement. In general, drivers increased the proportion of onroad glances as they approached the disengagement point. During the transition phase (i.e., in the $5 \mathrm{~s}$ around the disengagement), the 
Table 1: Average proportion (and standard deviation) of glance location during the interval of estimated steady-state driving with Autopilot (AP; i.e., the $-20 \mathrm{~s}$ to $-15 \mathrm{~s}$ interval before the disengagement) and the interval of estimated steady-state manual driving (i.e., in the $5 \mathrm{~s}$ to $10 \mathrm{~s}$ interval after the disengagement)

\begin{tabular}{lcc}
\hline Glance location & AP \% (sd) & Manual \% (sd) \\
\hline Road & $64(1.6)$ & $76(2.7)$ \\
Down center stack & $17(1.9)$ & $8(1.8)$ \\
Instrument cluster & $6(1)$ & $6(0.6)$ \\
Windows mirrors & $6(1.5)$ & $4(0.8)$ \\
Rearview mirror & $3(1)$ & $4(0.8)$ \\
Not available & $2(0.5)$ & $2(0.5)$ \\
Other & $2(0.7)$ & $0(0.3)$ \\
\hline
\end{tabular}

proportion of on-road glances increased from $72 \%$ to $82 \%$ at the time of the disengagement. At the end of the transition phase, drivers had about as much attention on the road $(76 \%)$ as during manual driving that follows. Conversely, the trend of off-road glances to the down and towards the center stack areas decreased while approaching the transition point. At the start of the transition phase, the proportion of glances down and to center stack was $12 \%$, but this value decreased to $2 \%$ at the time of disengagement. After the disengagement, this proportion increased to $5 \%$, and this value was maintained for the rest of the epoch.

While glances down and to the center stack areas accounted for most of the off-road glances at the beginning of the transition phase, glances to the instrument cluster were the most frequent at the disengagement point (6\%) and glances to the lateral windows and mirrors were the dominant at the end of the transition phase (9\%). Glances to other off-road glance areas were generally steady around the disengagement (Figure 4).

\subsection{Steering wheel control behavior}

Table 2: Average proportion (and standard deviation) of steering wheel control levels during the interval of estimated steady-state driving with Autopilot (AP; i.e., the -20 $s$ to $-15 s$ interval before the disengagement) and the interval of estimated steady-state manual driving (i.e., in the $5 \mathrm{~s}$ to $10 \mathrm{~s}$ interval after the disengagement).

\begin{tabular}{lcc}
\hline Steering wheel control level & AP \% (sd) & Manual \% (sd) \\
\hline High & $4(0.2)$ & $11(0.4)$ \\
Medium & $2(0.3)$ & $18(0.4)$ \\
Low & $60(1)$ & $70(0.7)$ \\
None & $33(0.6)$ & $1(0.2)$ \\
Other & $2(0.3)$ & $0(0)$ \\
Not available & $0(0)$ & $0(0)$ \\
\hline
\end{tabular}

Steady-state steering wheel control behavior. Similar to what we did with glance behavior, Table 2 shows the aggregate steady-state steering wheel control levels with and without AP active. In general, drivers maintained a low level of steering wheel control in most of the events, regardless of AP use. However, the other categories of steering wheel control were opposite when comparing steadystate behavior before and after the disengagement. Notably, the proportion of event in which drivers did not hold the steering wheel was prominent with AP active and almost completely disappeared when drivers regained manual control.

Time-course of steering wheel control behavior. Figure 5 shows the proportion of levels of steering wheel control from $20 \mathrm{~s}$ before to 10 $\mathrm{s}$ after the AP disengagement. During the transition phase (i.e., in the $5 \mathrm{~s}$ around the disengagement), drivers' level of steering control changed dramatically. While at the beginning of the transition phase the proportion of no level of steering wheel control was still about $29 \%$, it dropped at the transition point (5\%) and it continued to decrease to $1 \%$ after the transition phase. This value was maintained for the remaining of the event. At the same time, the proportion of drivers with their hands placed on higher control of the steering wheel increased as they approached the disengagement point. Over the course of the transition, low control increased from $58 \%$ to $66 \%$; medium control increased from $6 \%$ to $19 \%$; high control increased from $6 \%$ to $13 \%$.

\section{DISCUSSION}

Based on the sample characteristics, AP was used extensively in this subsample of highway driving. The findings of this study open a window onto how actual Tesla users behave in relation to semiautomated assistive driving technology. Among all driver-initiated transitions, the transitions between AP and manual driving were the most frequent, suggesting that drivers preferred to use AP over T-ACC alone (Figure 3). Because the data in this study are drawn from a sample of Tesla owners, driving their own vehicles, the observations provide insights on more established effects of vehicle automation on driver behavior. Until now, what we know about the use of AP in real-world traffic comes from relatively small scale onroad studies $[1,9,10]$, where participants likely had less experience with AP.

\subsection{Glance behavior}

The observed general decrease in on-road glances when using AP compared to manual driving prior to and following AP disengagement is consistent with findings from previous naturalistic studies [40]. While in manual driving the proportion of on-road glances is generally about $85 \%$ [41], when driving with ACC the proportion of on-road glances are around $78 \%$ [40], and in the current study, we find that the on-road glances with AP is lower, averaging around $64 \%$. Considering these trends, the decrease in on-road glances with the increase in automation level is likely to be associated with a reduction in overt driving task demand (moment-to-moment engagement necessary to actively keep the vehicle at a set-speed and spacing from the vehicle ahead, due to ACC, and positioned in the lane, due to Autosteer) and perhaps an increase in complacent reliance on the system. Our results suggest that AP may have a greater impact on attention to the road than the $74 \%$ proportion of on-road glances with AP previously reported by Gaspar and Carney [15], where the impact of AP was considered in a more limited exposure (1 week) sample. Contrary to the results in Gaspar 


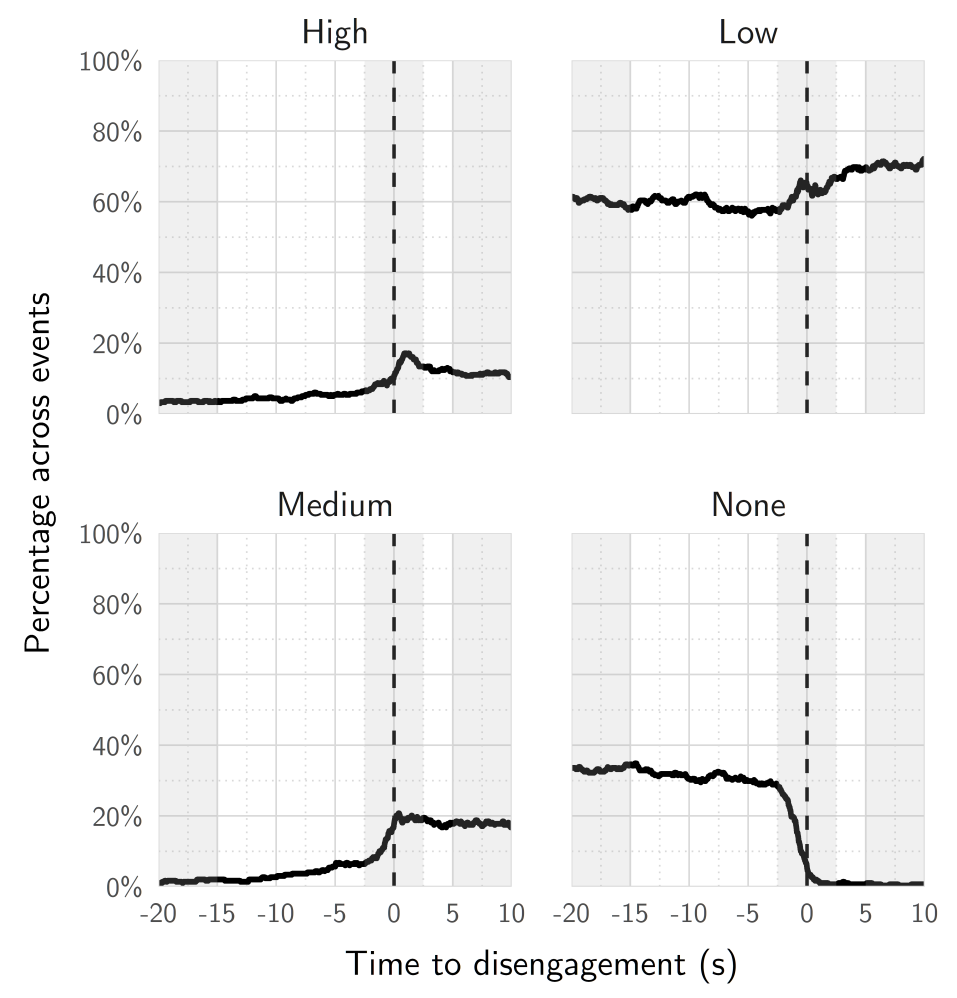

Figure 5: Proportion of steering wheel control levels at any given time during the events under analysis. AP disengagement is at $0 \mathrm{~s}$, marked with black dashed line. The shaded bands indicate the interval of estimated steady-state driving with Autopilot (AP; i.e., the $-20 \mathrm{~s}$ to $-15 \mathrm{~s}$ interval before the disengagement), the transition phase from AP to manual driving (i.e., the $-2.5 \mathrm{~s}$ to $2.5 \mathrm{~s}$ interval around the disengagement) and the interval of estimated steady-state manual driving (i.e., the $5 \mathrm{~s}$ to $10 \mathrm{~s}$ interval after the disengagement).

and Carney [15], we found that the decrease in on-road glances with AP corresponded with a noticeable increase of glances down and to center stack. Because drivers may glance down when using a smartphone, or glance to center stack while interacting with the touchscreen, one possible explanation is an increase in secondary task engagement with AP compared to manual driving-a trend that was previously found in simulator studies [2,17]. At this stage, we have not coded information on secondary task engagement for Tesla participants, so a quantitative understanding of attention away from the road will be a focus of future work.

Attention changes cannot be considered in isolation of the potential safety benefits of AP and the bundle of other active-safety systems. By maintaining the vehicle in the lane and at a safe distance from other vehicles, and reducing the overall demand of driving, AP may reduce the overall exposure to critical situations $[16,35]$ and increase the frequency of passive driving behaviors such as car following. It remains unclear what is the appropriate driver attention level that meets the safety standard of a manually controlled vehicle, or if the safety standard of a manually controlled vehicle is an acceptable benchmark (e.g., in the case of the NHTSA Driver Distraction Guidelines [25]). Future work will need to comprehensively consider, in statistically controlled analysis, the net safety benefits of systems like AP in relation to changes in risk exposure.

We found that the proportion of on-road glances during steadystate manual driving ( $76 \%$ in the $5 \mathrm{~s}$ to $10 \mathrm{~s}$ interval following transition in Figure 4; see also Table 1) remains lower than existing reference values for manual driving (85\% [41]) but similar to the value found with ACC (78\% [40]). This difference may be due to carryover effects of the automation or different driving scenarios across studies. For example, when drivers disengaged AP to change lanes and take the exit ramp, they may have checked the vehicle surroundings (as shown by the increase, albeit slight, of glances to the lateral windows and mirror after the transition to manual driving, Figure 4) or checked the navigation system for directions (as shown by the slight increase of glances to the center stack towards the end of the event, Figure 4). Some off-road glances (e.g., those to the side mirrors or to the instrument cluster) may be critical for safety, but off-road glances farther away from the forward pathway towards non-driving related areas may increase risk. Future work may consider contextual aspects associated with these glance behavior patterns.

Drivers' attention to the road appears to increase and attention down and away from the road decreases as they approach the point of a driver-initiated automation disengagement. While additional 
data following the coding window are needed to confirm any trend, the plots suggest a brief leveling of both metrics following the transition with indications of re-engagement of downward glances to follow. These observations are suggestive of behaviors that one would expect to occur in the lead-up to a planned action and the type of recovery of awareness to follow. As such, the data provide some confidence in the fact that the transitions may be more likely to be planned then emergent. More detailed analysis of individual transitions may be a core component of a more comprehensive statistical consideration of the data.

\subsection{Steering wheel control level}

There is little quantitative information on how drivers maintain control of the steering wheel during manual or automated driving [e.g., 33]. Adequate control of the steering wheel is critical both to correct lateral position in normal driving and to evade emergency situations. The study by Walton and Thomas [43] is one of the few on driver hand placements in naturalistic manual driving. Walton and Thomas [43] observed that drivers kept at least one hand on the top-half of the steering wheel in most of the cases. The current analysis found that, in most of the events, the Tesla drivers had low steering wheel control, independently of AP use.

Walton and Thomas [43] argued that driver hand position was likely a comfort decision. In fact, they noticed drivers regaining a higher level of control when the task demand increased (e.g., at higher speed). In general, we found that drivers had a lower level of control of the steering wheel with AP compared to manual driving. One of the benefits of AP is to reduce the physical effort of controlling the vehicle compared to manual driving. Thus, we would expect drivers to prefer keeping their hands at a more comfortable hand position (including driving hands-free) when steering is automated. At the time of transition, there was an uptick in both medium and high level of control, which may indicate that drivers actively chose to regain a higher control of the vehicle to perform a maneuver (e.g., lane change).

Few studies have examined driver hand positions with automation. In their simulator experiment, Piccinini et al. [30] did not find any striking difference in hands placement between driving with and without ACC active. Moreover, Piccinini et al. [30] found that drivers generally adopted higher control positions (e.g., the proportion of high level of control was above $40 \%$ regardless of ACC use [30]). In contrast, we found major differences. The most striking difference was in a higher proportion of the drivers not holding the steering wheel with AP compared to manual driving (Table 2). This finding is in line with the notion that drivers seek comfort and may take advantage of automation to get it.

In their open-road study with AP, Banks et al. [1] did not specifically reported on hands placement, but they found that most participants received frequent hand-on-wheel warnings. This indicates a tendency, even for inexperienced AP users, to release control of the steering wheel when possible. In their test-track experiment with a system similar to AP, Victor et al. [42] found that participants tended to take their hands off the wheel. The $39 \%$ proportion of no steering control $20 \mathrm{~s}$ prior to the disengagement further supports the findings by Banks et al. [1] and Victor et al. [42], and suggests that many operators are leveraging AP in conflict with hands on the wheel guidance provided in the vehicle owner's manual [37]. The steady decrease of glances down and to the center stack in the $20 \mathrm{~s}$ before the AP disengagement, while road glances steadily increased, suggests that drivers progressively redirected attention to the driving scenario before the transition. Drivers seem to move their hands to the wheel closer to the point of disengagement. The three intervals we identified (steady-state driving with AP, transition phase, and steady-state manual driving) suggest that attention shifts begin on average in advance of actions associated with moving hands towards a higher level of control. More research is needed to examine the relationship of the response process (glance and steering control behavior) in relation to the driving context of transitions.

It remains an open question as to how drivers prepared and planned the transition of control. Previous research suggested that vestibular-kinesthetic-somatosensory information (for example, perceptual cues that originate from the vehicle modulating speed) may alert drivers of an impending conflict [23]. As the events included in this paper were not critical, it is unclear what cues (if any) elicited drivers' visual and motor response. Perhaps, drivers increased their engagement to the driving task based upon the upcoming driving context [39]. For example, drivers may have been, in some instances, familiar with the route and prepared to regain control in anticipation of an exit ramp. On one hand, AP may be beneficial in allowing drivers to monitor the surroundings throughout the drive without the need to control the vehicle. On the other hand, if drivers uptake a visual and manual demanding secondary task, their awareness of the changing traffic situation may decrease rapidly [9] and extra time may be needed to successfully regain control of the steering wheel. Finally, it is unclear how drivers that exhibit a decrease of attention and steering control may cope with system failures that do not generate vestibular-kinestheticsomatosensory cues (e.g., lane drifts).

\subsection{Limitations and future directions}

The data used in this paper were collected prior to an AP software update (v. 2018.21.9) that is described to increase requirements for drivers to hold the steering wheel. As such, the effects of this software update on driver behavior are a topic of future study. Driving context was not investigated, although driver visual response has been shown to depend on, for example, the presence of a lead vehicle [24]. Future research could study the interplay between driver behavior and the complexity of the traffic situation. Future work may also consider different types of and reasons for disengagement (e.g., system-initiated by using a larger sample than the one leveraged in this analysis). Furthermore, as increases in inattention and distraction may become more pronounced after prolonged periods of automated driving, future work may examine change of behavior over time as many of the drivers in our study have been under observation for several years. Finally, this paper presents initial exploratory results. In the future, we intend to expand on these results with statistical modeling (e.g. Bayesian statistics).

\section{CONCLUSIONS}

This work provides new objective data on the naturalistic utilization of AP through shifts in attention and hand positions during driverinitiated transitions of control. These findings begin to enhance 
society's understanding of the use of AP in real-world conditions. The intended use of AP, as specified in the user's manual, requires drivers to remain attentive and to keep their hands on the steering wheel. Data in this paper suggests that some drivers are not using $\mathrm{AP}$ in a manner consistent with recommendations of the manufacturer. The findings support the call for increased management of driver attention to meet manufacturer recommended use $[20,21,32]$. What is not clear from this data, however, is if newer software updates have remediated the observed behavior. With AP active, we found that, in general, (i) drivers pay more attention to the road in the seconds prior to and after disengaging AP; (ii) most of the off-road glances during AP use were downwards and to the center-stack, which may indicate decreased driving related attention and increased engagement with secondary tasks; (iii) drivers often have low control of the steering wheel or drive hands-free. Overall, these findings suggest that drivers are less attentive to the driving task when AP is active compared to when driving following AP disengagement-it remains an open question as to how attentive or engaged a driver must be with the support of an assistive driving system, such as AP, to meet or exceed the safety threshold of a conventional vehicle. It is also debated how much safer automated vehicles need to be before being introduced on public roads [19]. For example, introducing an automated vehicle with a safety performance slightly better ( $10 \%$ better) than the average driver may save overall more lives than waiting for a nearly perfect $(90 \%$ better) system [18]. As the performance of automation depends on the interaction between the human and the system, we hope that the data provides information to designers charged with developing assistive and automated systems and empowers regulators and safety advocates with data needed to better objectively assess how to guide appropriate utilization of such technology.

\section{ACKNOWLEDGMENTS}

Data were drawn from work supported by the Advanced Vehicle Technology (AVT) Consortium. The first author was supported by the Santos Family Foundation. The co-authors were supported by the AVT Consortium. The views and conclusions being expressed are those of the authors and have not been sponsored, approved, or endorsed by the Consortium or Foundation. The authors would like to thank colleagues at the MIT AgeLab, members in the AVT consortium, and three anonymous reviewers for their comments and suggestions on this paper.

\section{REFERENCES}

[1] Victoria A. Banks, Alexander Eriksson, Jim O’Donoghue, and Neville A. Stanton 2018. Is partially automated driving a bad idea? Observations from an on-road study. Applied Ergonomics 68 (2018), 138-145. https://doi.org/10.1016/j.apergo. 2017.11.010

[2] Oliver Carsten, Frank Lai, Yvonne Barnard, A. Hamish Jamson, and Natasha Merat. 2012. Control Task Substitution in Semiautomated Driving Does It Matter What Aspects Are Automated? Human Factors: The fournal of the Human Factors and Ergonomics Society (2012), 0018720812460246-0018720812460246.

[3] commaai. 2020. openDBC. https://github.com/commaai/opendbc

[4] Consumer Report. 2018. Ranking of Automated Driving Systems. (2018) https://www.consumerreports.org/autonomous-driving/cadillac-tops-tesla-inautomated-systems-ranking/

[5] Dick De Waard, Thigri Van den Bold, and Ben Lewis-Evans. 2010. Driver hand position on the steering wheel while merging into motorway traffic. Transportation Research Part F: Traffic Psychology and Behaviour 13, 2 (2010), 129-140. https://doi.org/10.1016/j.trf.2009.12.003
[6] Joost C. F. de Winter, Riender Happee, Marieke H. Martens, and Neville A. Stanton. 2014. Effects of adaptive cruise control and highly automated driving on workload and situation awareness: A review of the empirical evidence. Transportation Research Part F: Traffic Psychology and Behaviour 27 (2014), 196-217. https: //doi.org/10.1016/j.trf.2014.06.016

[7] Murat Dikmen and Catherine Burns. 2017. Trust in autonomous vehicles: The case of tesla autopilot and summon. In 2017 IEEE International Conference on Systems, Man, and Cybernetics (SMC). IEEE, Banff (Canada), 1093-1098.

[8] Murat Dikmen and Catherine M Burns. 2016. Autonomous driving in the real world: Experiences with tesla autopilot and summon. In Proceedings of the 8th international conference on automotive user interfaces and interactive vehicular applications. ACM, Ann Arbor, MI, USA, 225-228.

[9] Mica R Endsley. 2017. Autonomous driving systems: A preliminary naturalistic study of the Tesla Model S. Fournal of Cognitive Engineering and Decision Making 11, 3 (2017), 225-238.

[10] Alexander Eriksson, Victoria A. Banks, and Neville A. Stanton. 2017. Transition to manual: Comparing simulator with on-road control transitions. Accident Analysis \& Prevention 102 (2017), 227-234. https://doi.org/10.1016/j.aap.2017.03.011

[11] Alexander Eriksson and Neville A Stanton. 2017. Takeover time in highly automated vehicles: noncritical transitions to and from manual control. Human factors 59, 4 (2017), 689-705

[12] Euro NCAP. 2018. 2018 Automated Driving Tests - Tesla Model S. (2018). https://cdn.euroncap.com/media/41590/euro-ncap-automated-driving2018-tesla-model-s-datasheet.201810171432105270.pdf

[13] Jack Ewing. 2020. German Court Says Tesla Self-Driving Claims Are Misleading. The New York Times (2020). https://www.nytimes.com/2020/07/14/business/teslaautopilot-germany.html

[14] Lex Fridman, Daniel E. Brown, Michael Glazer, William Angell, Spencer Dodd, Benedikt Jenik, Jack Terwilliger, Aleksandr Patsekin, Julia Kindelsberger, Li Ding, Sean Seaman, Alea Mehler, Andrew Sipperley, Anthony Pettinato, Bobbie D. Seppelt, Linda Angell, Bruce Mehler, and Bryan Reimer. 2019. MIT Advanced Vehicle Technology Study: Large-Scale Naturalistic Driving Study of Driver Behavior and Interaction With Automation. IEEE Access 7 (2019), 102021-102038. https://doi.org/10.1109/ACCESS.2019.2926040

[15] John Gaspar and Cher Carney. 2019. The Effect of Partial Automation on Driver Attention: A Naturalistic Driving Study. Human Factors 61, 8 (2019), 1261-1276. https://doi.org/10.1177/0018720819836310

[16] Pnina Gershon, Charles Green, Bruce Mehler, and Bryan Reimer. 2021. The impact of automation on elevated driving kinematics. In The 7th International Conference on Traffic \& Transport Psychology. Gothenburg (Sweden).

[17] A. Hamish Jamson, Natasha Merat, Oliver M. J. Carsten, and Frank C. H. Lai. 2013. Behavioural changes in drivers experiencing highly-automated vehicle control in varying traffic conditions. Transportation Research Part C: Emerging Technologies 30 (2013), 116-125. https://doi.org/10.1016/j.trc.2013.02.008

[18] Nidhi Kalra and David G. Groves. 2017. The Enemy of Good: Estimating the Cost of Waiting for Nearly Perfect Automated Vehicles. Report. Rand corp. https: //www.rand.org/pubs/research reports/RR2150.html

[19] Magdalena Lindman, Irene Isaksson-Hellman, and Johan Strandroth. 2017. Basic numbers needed to understand the traffic safety effect of automated cars. In IRCOBI Conference 2017. Antwerp (Belgium), 1-12. https://www.ircobi.org/ wordpress/downloads/irc17/pdf-files/10.pdf

[20] Edward J. Markey. 2019. Senator Markey urges Tesla to address safety flaws in its Autopilot system. https://www.markey.senate.gov/news/press-releases/senatormarkey-urges-tesla-to-address-safety-flaws-in-its-autopilot-system

[21] Edward J. Markey and Richard Blumenthal. 2020. Stay Aware For Everyone (SAFE) Act of 2020. https://www.markey.senate.gov/imo/media/doc/SAFE\%20Act.pdf 116th Congress, 2d Session.

[22] Callum D. Mole, Otto Lappi, Oscar Giles, Gustav Markkula, Franck Mars, and Richard M. Wilkie. 2019. Getting Back Into the Loop: The Perceptual-Motor Determinants of Successful Transitions out of Automated Driving. Human Factors 61, 7 (2019), 1037-1065. https://doi.org/10.1177/0018720819829594

[23] Alberto Morando, Trent Victor, and Marco Dozza. 2016. Drivers anticipate leadvehicle conflicts during automated longitudinal control: Sensory cues capture driver attention and promote appropriate and timely responses. Accident Analysis \& Prevention 97 (2016), 206-219. https://doi.org/10.1016/j.aap.2016.08.025

[24] Alberto Morando, Trent Victor, and Marco Dozza. 2019. A Reference Model for Driver Attention in Automation: Glance Behavior Changes During Lateral and Longitudinal Assistance. IEEE Transactions on Intelligent Transportation Systems 20, 8 (2019), 2999-3009. https://doi.org/10.1109/TITS.2018.2870909

[25] National Highway Traffic Safety Administration (NHTSA). 2013. Visual-Manual NHTSA Driver Distraction Guidelines for In-Vehicle Electronic Devices (Docket No. NHTSA-2010-0053). Report Federal Register vol. 78, no. 81, p. 24818-24890. National Highway Traffic Safety Administration. https://www.gpo.gov/fdsys/ pkg/FR-2013-04-26/pdf/2013-09883.pdf

[26] National Transportation Safety Board (NTSB). 2020. Collision Between a Sport Utility Vehicle Operating With Partial Driving Automation and a Crash Attenuator. Report NTSB/HAR-20/01 PB2020-100112. National Transportation Safety Board (NTSB). 
[27] National Transportation Safety Board (NTSB). 2020. Collision Between Car Operating with Partial Driving Automation and Truck-Tractor Semitrailer. Report NTSB/HAB-20/01. National Transportation Safety Board (NTSB).

[28] Linda Onnasch, Christopher D. Wickens, Huiyang Li, and Dietrich Manzey. 2014 Human Performance Consequences of Stages and Levels of Automation:An Integrated Meta-Analysis. Human Factors 56, 3 (2014), 476-488. https://doi.org/ $10.1177 / 0018720813501549$

[29] Raja Parasuraman and Victor Riley. 1997. Humans and automation: Use, misuse, disuse, abuse. Human Factors 39, 2 (1997), 230-230.

[30] G. B. Piccinini, G. Prati, L. Pietrantoni, C. Manzini, C. M. Rodrigues, and M. Leitão 2013. Drivers' hand positions on the steering wheel while using Adaptive Cruise Control (ACC) and driving without the system. Proceedings of the Human Factors and Ergonomics Society Europe (2013), 207-216.

[31] Quality Control Systems Corp. 2019. NHTSA's Implausible Safety Claim for Tesla's Autosteer Driver Assistance System. Report. Quality Control Systems Corp. https://www.safetyresearch.net/Library/NHTSA_Autosteer_Safety_Claim.pdf

[32] Bryan Reimer. 2020. Should Tesla Take The Initiative To Better Monitor And Manage Driver Behavior With Autopilot? Forbes (2020).

[33] Bryan Reimer, Anthony Pettinato, Lex Fridman, Joonbum Lee, Bruce Mehler, Bobbie Seppelt, Junghee Park, and Karl Iagnemma. 2016. Behavioral impact of drivers' roles in automated driving. In Proceedings of the 8th International Conference on Automotive User Interfaces and Interactive Vehicular Applications. ACM, Ann Arbor, MI, USA, 217-224.

[34] SAE. 2018. Taxonomy and definitions for terms related to on-road motor vehicle automated driving systems (j3016). https://saemobilus.sae.org/content/j3016_ 201806

[35] Bobbie D. Seppelt and Trent Victor. 2016. Potential Solutions to Human Factors Challenges in Road Vehicle Automation. Springer International Publishing, Cham,
Book section Chapter 11, 131-148. https://doi.org/10.1007/978-3-319-40503-2 11

[36] Alison Smiley. 2000. Behavioral Adaptation, Safety, and Intelligent Transportation Systems. Transportation Research Record: Fournal of the Transportation Research Board 1724 (2000), 47-51. https://doi.org/10.3141/1724-07

[37] Tesla. 2019. Model S owner's manual.

[38] Tesla. 2020. Vehicle safety report. https://www.tesla.com/VehicleSafetyRepor Accessed: May 2020.

[39] Emma Tivesten and Marco Dozza. 2015. Driving context influences drivers' decision to engage in visual-manual phone tasks: Evidence from a naturalistic driving study. Fournal of Safety Research 53 (2015), 87-96.

[40] Emma Tivesten, Alberto Morando, and Trent Victor. 2015. The timecourse of driver visual attention in naturalistic driving with adaptive cruise control and forward collision warning. In International Conference on Driver Distraction and Inattention, 4th. ARRB, Sydney, New South Wales, Australia, 1-14.

[41] Trent Victor, Marco Dozza, Jonas Bärgman, Christian-Nils Boda, Johan Engström, Carol Flannagan, John D. Lee, and Gustav Markkula. 2015. SHRP2 - Analysis of naturalistic driving study data: Safer glances, driver inattention and crash risk. Report. Transportation Research Board, 500 Fifth St. N.W., Washington, DC 20001, United States. http://www.trb.org/Publications/PubsSHRP2ResearchReportsSafety.aspx

[42] Trent Victor, Emma Tivesten, Pär Gustavsson, Joel Johansson, Fredrik Sangberg, and Mikael Ljung Aust. 2018. Automation Expectation Mismatch: Incorrect Prediction Despite Eyes on Threat and Hands on Wheel. Human Factors 60, 8 (2018), 1095-1116. https://doi.org/10.1177/0018720818788164

[43] Darren Walton and Jared A. Thomas. 2005. Naturalistic observations of driver hand positions. Transportation Research Part F: Traffic Psychology and Behaviour 8, 3 (2005), 229-238. https://doi.org/10.1016/j.trf.2005.04.010 